\title{
FITTING THE LUMINOSITY DECAY IN THE TEVATRON*
}

\author{
E. McCrory, V. Shiltsev, A. J. Slaughter, A. Xiao; Fermilab, Batavia, IL, U.S.A..
}

\section{Abstract}

This paper explores how to fit the decay of the luminosity in the Tevatron. The standard assumptions of a fixed-lifetime exponential decay are only appropriate for very short time intervals. A "1/time" functional form fits well, and is supported by analytical derivations. A more complex form, assuming a time-varying lifetime-like term, also produces good results. Changes in the luminosity can be factored into two phenomena: The luminosity burn-off rate, and the burn-off rate from nonluminosity effects. This is particularly relevant for the antiprotons in the Tevatron. The luminous and the nonluminous burn rate of the antiprotons are shown for Tevatron stores.

\section{INTRODUCTION}

This paper covers two related topics: (1) The functional form of the luminosity decay and (2) the luminous and non-luminous factors in the Tevatron luminosity decay.

The decay of the luminosity in the Tevatron is crucial to understand: The number of high-energy physics events seen by the experiments is directly proportional to their integrated luminosity. This integrated luminosity depends only on two factors: The luminosity at the beginning of a proton-antiproton store, and the way in which the luminosity decays away. The functional form of this decay is described here.

A simple exponential decay fits the luminosity decay poorly. But given the wide acceptability of this functional form, it the accepted fit form at Fermilab. In order to use this form, fits are restricted to two hours.

This paper explores two different assumptions for the luminosity decay. First, an exponential form is assumed, but with a time-dependent lifetime-like term within the exponential. The second, based on the analysis of our companion paper [1], has a functional form of one-overtime. Both functional forms work well, much better than a simple exponential decay.

Armed with an effective way to characterize the luminosity decay, it is possible to calculate for any store the relative contribution of the change in the antiproton intensity due to the luminosity (called the "luminosity burn off') to the decay that is not due to burn off. The last part of this paper analyzes this.

\section{FUNCTIONAL FORMS}

These three functional forms of the luminosity evolution are considered.

$$
\begin{aligned}
& \mathfrak{L}(t)=\mathfrak{L}(0) \exp (-t / \tau) \\
& \mathfrak{L}(t)=\mathfrak{L}(0) \exp (-t / T(t)) \\
& \mathfrak{L}(t)=\mathfrak{L}(0) /\left(1+t /\left(\tau_{0} C_{3}\right)\right)^{\mathrm{x}(t)}
\end{aligned}
$$

*Operated by Universities Research Association Inc. under Contract No. DE-AC02-76CH03000 with the US Dept of Energy.
$T(t)$ in the second form is:

$$
T(t)=T_{0}+C_{1} * t^{C_{2}}
$$

The four parameters $\mathfrak{L}(0), T_{0}, \mathrm{C}_{1}$ and $\mathrm{C}_{2}$ are allowed to vary in each fit. The time dependence of the exponent in (3) is a simple two-parameter linear function of time:

$$
x(t)=C_{3}+C_{4} t
$$

(The luminosity decay that is used in the operational model [3] uses equation 2 because it gives the most accurate fits for the widest range of stores.)

A typical (good) store from early in 2005 (number 4081 ) is selected to show the quality of these fits. The Minuit software package [2] is used to perform the fits. See Figure 1. These fits have the following parameters:

Eq. $1: \mathfrak{L}(0): 80, \tau: 15.5, \chi^{2} / \mathrm{DOF}: 3517$

Eq. $2: \mathfrak{L}(0): 109, T_{0}: 2.2, \mathrm{C}_{1}: 2.3, \mathrm{C}_{2}: 0.525 \chi^{2} / \mathrm{DOF}: 48$

Eq. 3: $\mathscr{L}(0): 106, \tau_{0}: 4.3, \mathrm{C}_{3}: 0.53, \mathrm{C}_{4}: 7 \mathrm{E}-3, \chi^{2} / \mathrm{DOF}: 37$

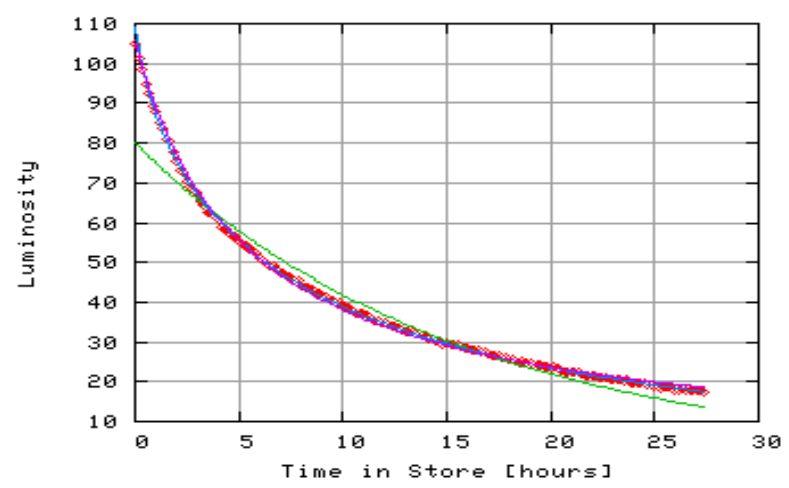

Figure 1: Minuit fit results for three types of functional forms: Green is (1); (2) and (3) are buried within the red data markers, for store \#4081. The luminosity is measured at the CDF experiment and is in the units $\left[\mathrm{cm}^{2} \mathrm{sec}\right]^{-1}$

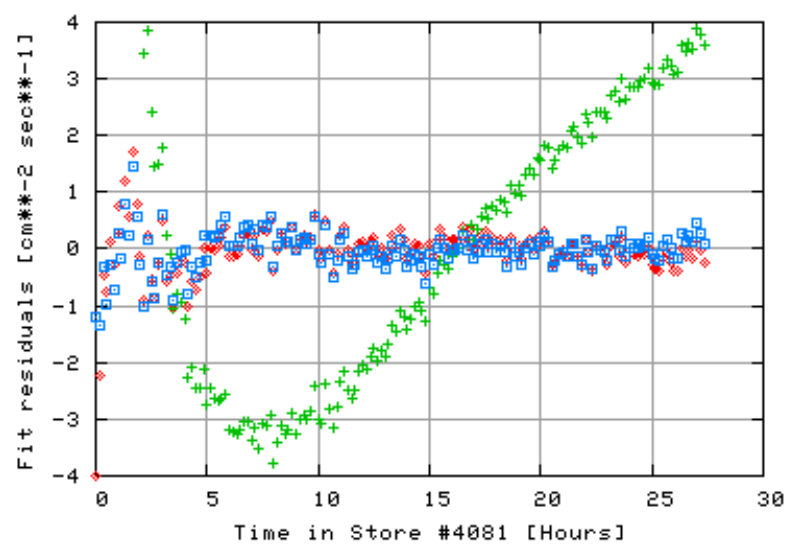

Figure 2: Fit residuals for store $\# 4081$.

It is obvious that the simple exponential form is not adequate. The fit residuals are shown in Figure 2; the residual for the exponential fit is about 25 at $\mathrm{t}=0$. The green plusses are the residuals for the simple exponential fit, equation 1; the red diamonds are for equation 2; and the blue squares are for equation 3 . 
The luminosity for each of the 36 individual bunch collisions in the Tevatron is also measured, and equivalent fits have been performed. Similarly, good fits are obtained from equations 2 and 3 .

The exponent to the denominator for the fits using equation 3 is derived to be unity [1]. A fit is performed where the exponent is allowed to vary, but is independent of time $\left(C_{4}=0\right)$. Figure 3 is the same as Figure 2 except only two fits are shown: for the fit to equation (3) with the time-dependent exponent (green plusses), and fits to equation (3) where the exponent is independent of time but allowed to float (red diamonds).

Again, assuming $C_{4}=0$, then for the stores from the past 12 months, we get the time-independent exponent versus the store number, as shown in Figure 4.

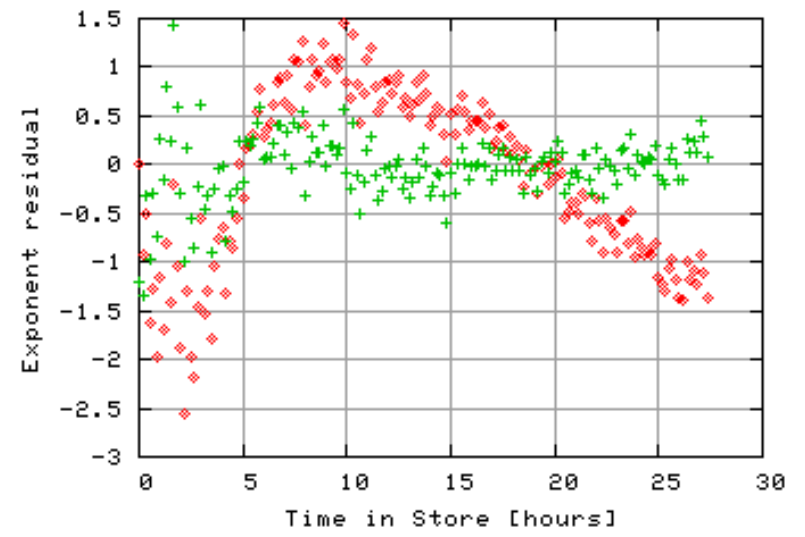

Figure 3: Comparing residuals for equation (3) with the exponent not time dependent (red diamonds) and time dependent (green plusses). The units on the vertical scale are $\left[\mathrm{cm}^{2} \mathrm{sec}\right]^{-1}$.

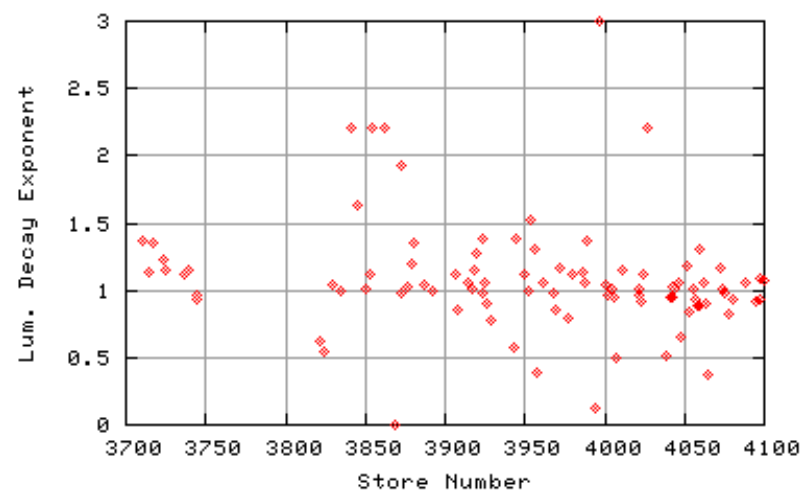

Figure 4: Initial value of the time-independent exponent to equation (3) versus store number (time).

There is clustering around unity, verifying the results of our companion paper [1].

\section{BURN RATE OF ANTIPROTONS}

The rate at which the antiprotons are removed ("burned up") from the Tevatron due to the luminosity is calculable directly from the total proton/antiproton total cross section:

$$
d N(A) / d t=-0.252 \times \Sigma \mathfrak{L}_{i}
$$

Here, the number of antiprotons, $N(A)$, is expressed in units of $10^{9}$ particles, and the sum of the luminosities over the experiments is expressed in unit of $\left(\mathrm{cm}^{2} \mathrm{sec}\right)^{-1}$. The value 0.252 is derived directly from the total protonantiproton cross section at $1.96 \mathrm{TeV}, 70$ millibarns. The summation in equation 4 is over the two experiments in the Tevatron: CDF and D0.

The Tevatron antiproton bunch we call " 1 " collides with the proton bunch we call "13" at CDF and with the proton bunch we call " 25 " at D0, etc. The luminosities coming from these individual bunch collisions are measured directly by the experiments.

Using functional form (3), the decay in the number of antiprotons in each bunch within the Tevatron is fit for recent stores in the Tevatron. From this fit, the change in the intensity of the antiproton bunch is calculated numerically at each moment. Given the luminosity measured at each experiment and the relationship given in equation 4, the change in the antiproton intensity that is due to luminosity burn-off is calculated. These two quantities are subtracted, leaving the change in the intensity that is not due to luminosity burn off.
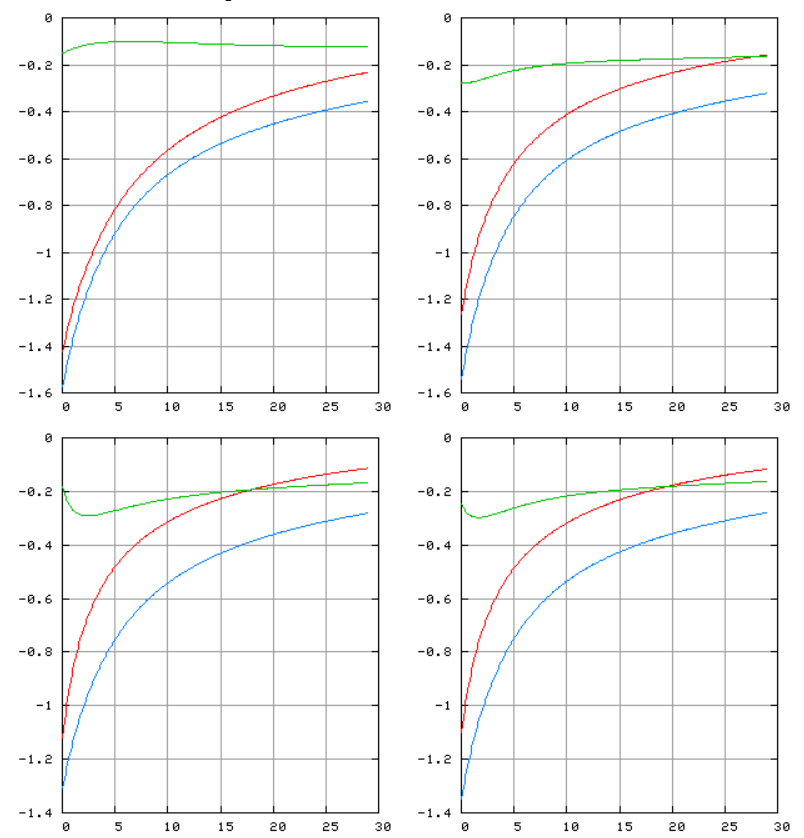

Figure 5: Antiproton intensity change [E10 / hour] for the collisions involving antiproton bunches number 1, 2, 3 and 4 versus time [hours] in store 3739.

This analysis is performed on the fitted functional form because the actual data are too noisy to produce clean results. A typical calculation for four of the 36 bunches within one store is show in Figure 5. These four charts are the intensity changes for antiproton bunch numbers 1 , 2,3 and 4 of store number 3739 . In each chart, the vertical scale has the units of intensity change per hour [(E10 particles)/hour]. The blue line in each chart is the change in the intensity per hour, calculated directly from the fit of the CDF luminosity data. The red line is the change in the intensity expected only from luminosity burn off, equation 4. The green line is the difference between the total 
intensity change and the intensity change that comes from only the luminosity burn-off. The error bars, as determined from the chi-squared per degree of freedom, are about equal to 0.1 units. These results are typical.

This analysis has been expanded to all bunches for several stores. Some patterns emerge. As shown in Figure 5, the dominant mechanism for losing antiprotons at the beginning of a store is through luminosity burn off. Also, during the course of a store the relative magnitude of the non-luminous burn off increases with respect to the total burn off. For most stores and bunches, the time at which the luminous and the non-luminous burn offs are equal is between 10 and 20 hours into the store.

These results can be seen equivalently as follows. Looking at these data from several stores, we calculate the fraction of the total burn off that is due to the luminosity and that is due to everything else. Figure 6 shows these fractions, averaged over the 36 bunches (with statistical errors) at the beginning of the store. Figure 7 is for 2 hours in and Figure 8 is for 10 hours in.

\section{CONCLUSIONS}

The decay of the luminosity is not described accurately by a simple exponential function. Good fits are obtained with a time-dependent lifetime-like term, or with a "oneover-time" functional form as described in our companion paper [1]. Either of these two functional forms provides adequate fits, both for the total luminosity or for the luminosity as measured bunch-by-bunch.

Using the fits results allows us, on average, to calculate the burn-off of the antiproton bunches in the Tevatron during the store and to determine the portion of this burnoff that is due to luminosity and that which is due to other non-productive effects. Most bunches in most stores initially have the bulk of their antiproton losses from luminosity burn-off. But by about 10 hours into a store, half of the antiproton burn-off is due to effects other than luminosity.

\section{REFERENCES}

[1] V. Shiltsev, E. McCrory, paper \#2497 (TPAP038), "Characterizing Luminosity Evolution in the Tevatron."

[2] F. James, M Roos, "Minuit: A system for Function Minimization and Analysis of Parameter Errors and Correlations," Computer Physics Communications, 10 (1975) 343.

[3] E. McCrory, paper \#1632 (TPAP037), "Monte Carlo of Tevatron Operations, Including the Recycler."

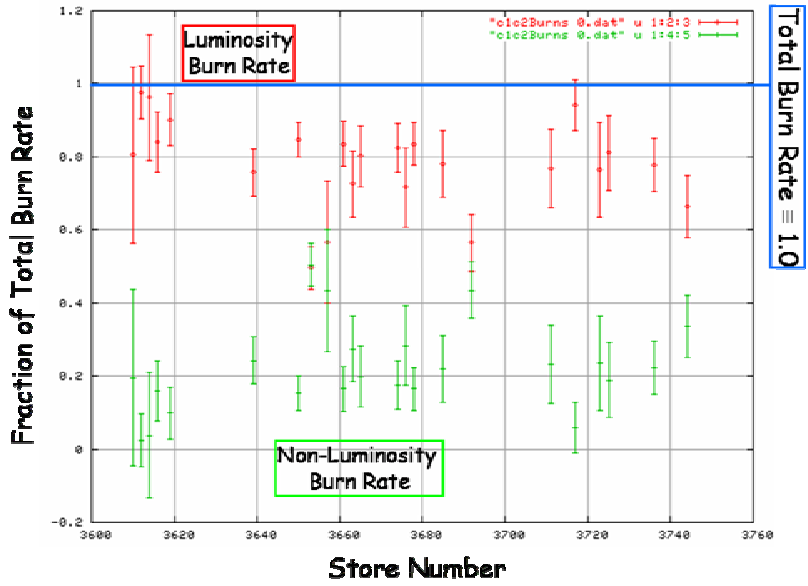

Figure 6: The fraction burn-off rate of the antiprotons at the beginning of several stores, averaged over all 36 bunches; Upper (red) points are the calculated change due to luminosity burn off; the lower (green) points are the calculated non-luminous burn-off.

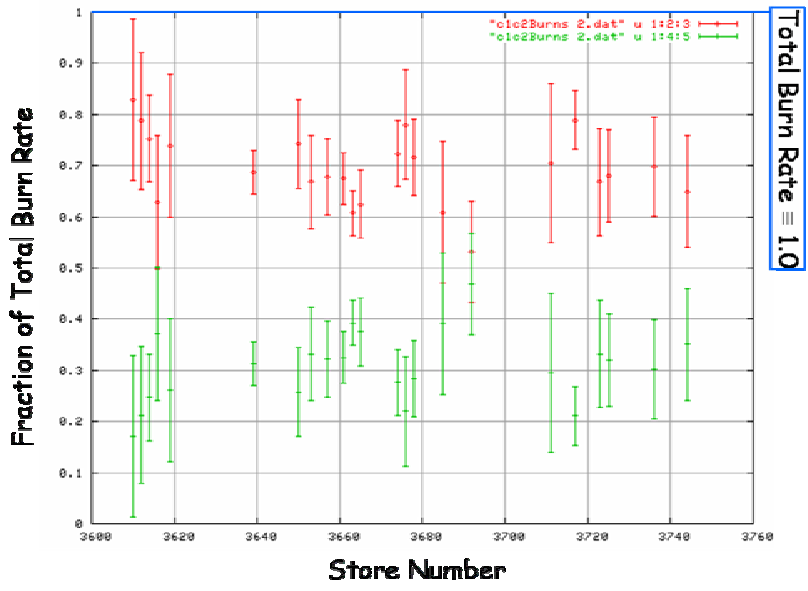

Figure 7: The fraction burn-off rate of the antiprotons at the 2-hour point of several stores.

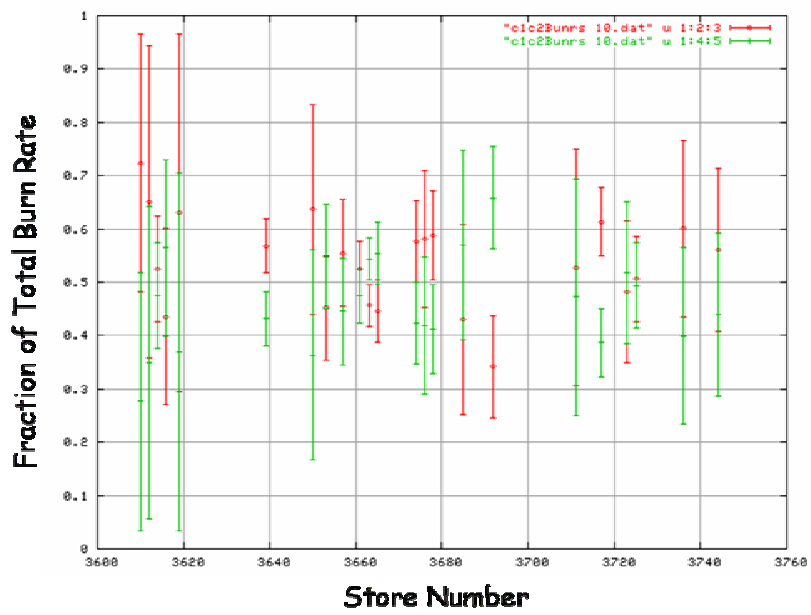

Figure 8: The fraction burn-off rate of the antiprotons at the 10-hour point of several stores. 